\title{
Smart Transportation System using IOT
}

\author{
Deeplaxmi V. Niture, Vivekanand Dhakane, Piyush Jawalkar, Ankit Bamnote
}

\begin{abstract}
In this paper a Smart Vehicle Assistance and Monitoring system (SVAMS) is presented. SVAMS is an intelligent transportation system (ITS), developed to tackle various traffic related issues. It is a traffic management, monitoring and optimization solution in which all the vehicles are interconnected through Zigbee and are monitored and assisted centrally, by a data center. The system has two parts; one part is mounted in/on the vehicle and the other part is at the data centre. Part one collects data from various sensors and transmits it to central data centre. All the data will be stored on cloud for further analysis, processing and future use. SVAMS is relatively low-cost, compact and has various functionalities such as emergency response, pollution level monitoring, automatic toll collection, traffic rule violation detection, vehicle tracking, etc. The use of SVAMS will help to build up Clean, Corruption free and Crime free (C-3) cities.
\end{abstract}

Keywords: Emergency services, message passing, vehicle safety, wireless mesh network, ZigBee.

\section{INTRODUCTION}

The rapid urbanization of cities in developing countries like India has resulted in a sharp rise in the number of vehicles on roads in recent years. As a consequence of which, there is an alarming raise of negative externality such as sever road congestion, air pollution, frequent road accident etc. which puts huge stress on the transportation system. Due to lack of adequate communication between vehicles themselves and vehicle-to-infrastructure there are lots of challenges in conventional traffic management solutions. The advancements in wireless communication and Internet capabilities have encouraged the development of ITS for improving traffic flow and safety. The latest developments in communication technology is one of the key factors for the effectiveness of ITS. The use of effective communication can help reduce congestion and gas emissions to a minimum level and it can also significantly improve the safety. However, in recent years, the Internet of Things (IoT) together with the

Manuscript received on June 20, 2021

Revised Manuscript received on June 26, 2021.

Manuscript published on June 30, 2021.

* Correspondence Author

Dr. Deeplaxmi V. Niture*, Department of Electronics and Telecommunication Engineering. College of Engineering Pune (COEP) Pune, India. Email: dvn.extc@coep.ac.in

Vivekanand Dhakane, Department of Electronics and Telecommunication Engineering. College of Engineering Pune (COEP) Pune, India. Email: dhakanevr13.extc@coep.ac.in

Piyush Jawalkar, Department of Electronics and Telecommunication Engineering. College of Engineering Pune (COEP) Pune, India. Email: jawalkarpv13.extc@coep.ac.in

Ankit Bamnote, Department of Electronics and Telecommunication Engineering. College of Engineering Pune (COEP) Pune, India. Email: bamnorean13.extc@coep.ac.in

(C) The Authors. Published by Blue Eyes Intelligence Engineering and Sciences Publication (BEIESP). This is an open access article under the CC BY-NC-ND license (http://creativecommons.org/licenses/by-nc-nd/4.0/) concept of the Vehicular Ad-hoc Networks (VANETs) has motivated researchers to rethink and redevelop ITS [1].

The use of electronics in automobile has made many tasks possible, which seemed mechanically impossible earlier. For example, use of artificial intelligence, accident alert systems, electronic toll collection systems and location tracking systems in vehicles. Even though there are lots of components common in these systems, they are available in market as separate systems with different protocols and they are expensive as well. Therefore, we have designed and developed a system, which has most of the key functions of these systems and is compact in size and highly cost effective as well. This system has a capacity to replace existing systems in the market.

The work carried out is presented in this paper in the following sequence: Section II explains the architecture of SVAM system. Section III gives some calculations. This is followed by section IV which discusses test and results. Finally, section V is conclusion.

\section{SYSTEM ARCHITECTURE}

Fig. 1 shows block diagram of the SVAM system. It gives the working of the system as a whole. Every vehicle in the SVAM system has ZigBee box and GPS box. Both communicate with each other through i2c protocol. Vehicle communicates with data center using ZigBee at $900 \mathrm{MHz}$ frequency band. ZigBee range is approximately $9 \mathrm{~km}$. Data center has datacenter box and computer for data logging. They are connected with Bluetooth, whereas Data center uploads data to cloud using Wi-Fi. Toll collection box communicates with vehicle through IR transreciever and with cloud through Wi-Fi.

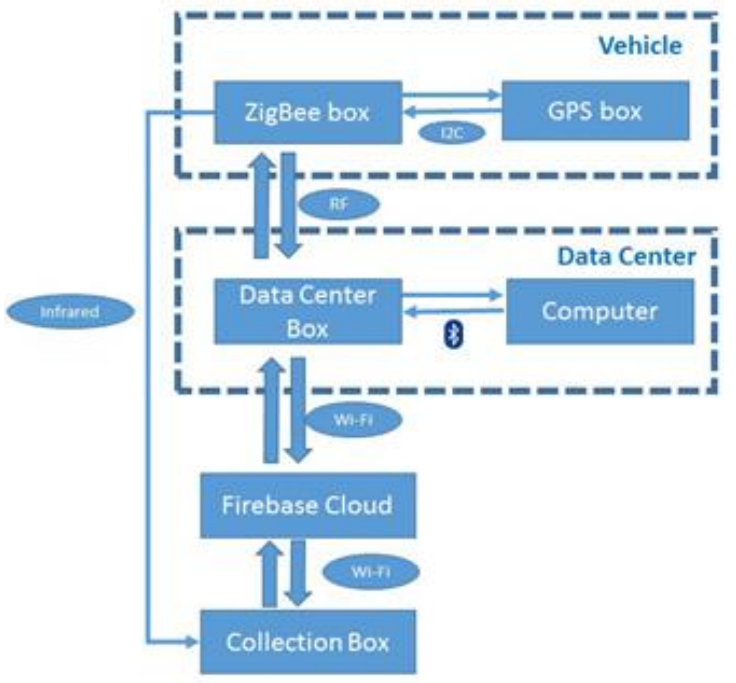

Fig. 1. Block Diagram of the SVAM System

Blue Eyes Intelligence Engineering and Sciences Publication 
The SVAM system perform following functions: Accident detection using Gyro meter, air pollution monitoring using MQ135, automatic toll collection using IR transreciever, detect rule violation using IR transreciever, location Tracking using GPS, centralized and easy accessible vehicle database using cloud, safe distance alert using ultrasonic distance sensor.

\section{A. Vehicle Module}

Vehicle module consists of a ZigBee box and a GPS box.

\section{ZigBee Box}

ZigBee box is designed to collect data from various sensors and from GPS box through i2c and transmit it to other vehicles or data center. Fig. 2 shows the schematic of ZigBee box. This box contains ZigBee, Atmega328p-pu, Antenna, Bluetooth HC-05 and Buck convertor PTN76060WAS.

IR transreceiver is mounted on top for communication with collection box for automatic toll collection and traffic rule violation detection. ZigBee has $10 \mathrm{~km}$ line of sight range [2].

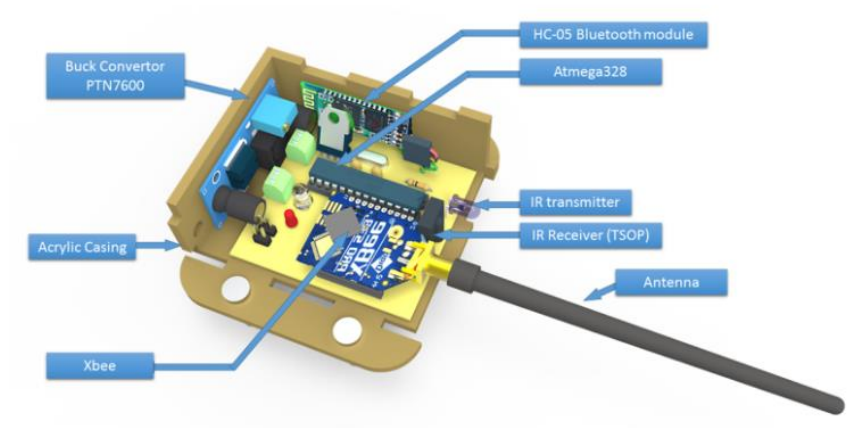

Fig. 2. ZigBee Box

\section{GPS box}

GPS box is another circuit (box) in vehicle, which is connected to ZigBee box through wire as both are in vehicle. Fig. 3 shows the GPS box. This box has components like GPS module, Serial LCD, two Atmega328 p-pu and MPU 6050 .

The GPS box has two micro-controllers. First microcontroller is for reading GPS and IMU. GPS module must connect to at least 4 satellites around the earth without having any obstacle in between [3]. For that it must be mounted with patch antenna which points upward without any metallic obstacle. When there is head on collision with other vehicle or any other obstacle (like tree, wall, etc), IMU gives acceleration and angle of vehicle to controller. Then by using the algorithm, the controller confirms the accident and communicates with ZigBee box. And subsequently ZigBee box sends message to nearby vehicles and ambulance. This requires only 6-7 milliseconds.

Second microcontroller is used for LCD, distance sensor and, Gas sensor monitoring. When there is any obstacle from backside or from front size, it will be detected by ultrasonic sensors and message will appear about obstacle on LCD. Gas sensor MQ 135 is used for hazardous gas level detection.

Gas sensor has heater inside to create condition in which gas can be detected. Resistance of this heater is $27 \Omega$ so it draws $=5 \mathrm{~V} / 27 \Omega=185 \mathrm{~mA}$ current. To avoid continuous flow of current through heater coil, it is controlled by using
MOSFET IRF510 whose gate is controlled by digital pin of controller.

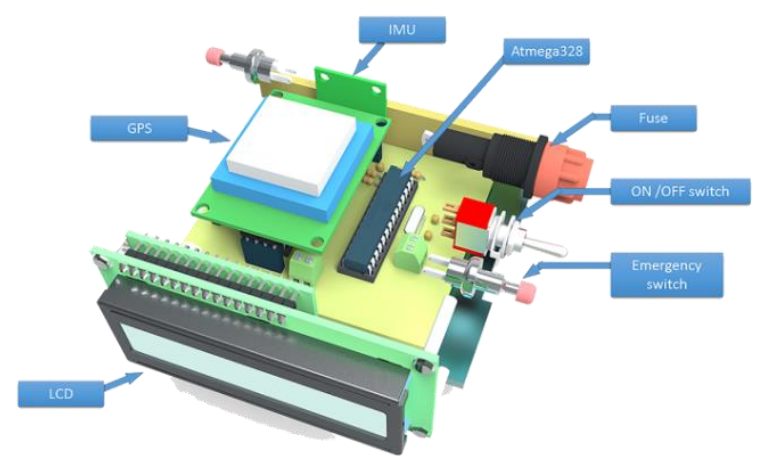

Fig. 3. GPS Box

\section{B. Data Center Module}

Data center module has a data center box and a computer/laptop with a software

\section{Computer Software at Data Center}

At data Centre, software designed in processing language -Low level java language is monitor for vehicles in SAVM system [4]. For designing Graphics User Interface G4P library is used in processing [5]. This software gets data from ZigBees as well as from firebase. It displays data about every vehicle. Fig. 4 shows the actual GUI of the software.

Important objects, numbered from 1 to 7 are: Message window for messaging vehicle, setting window for changing map style, track window to get details of vehicle by its number, no. of received and transmitted data packets, list of vehicle connected, select serial port of computer, and animated Logo.

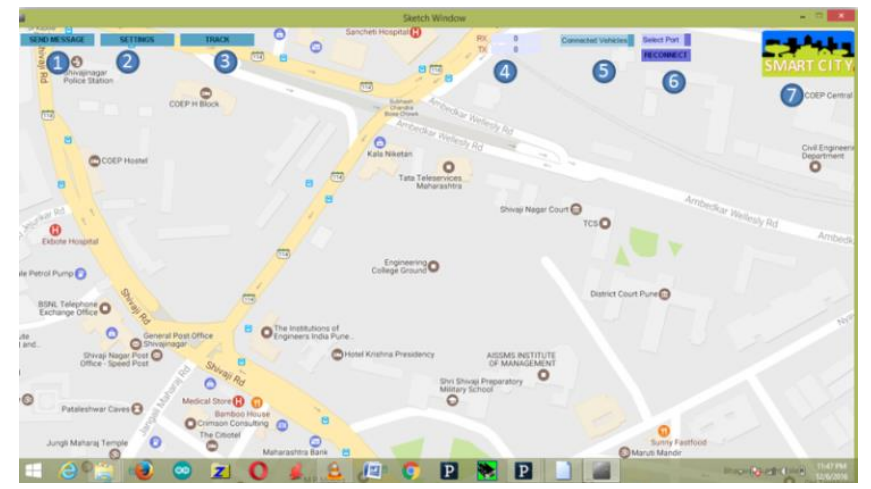

Fig. 4. Data centre Software GUI

\section{Data Center Box}

Data center box is circuit at Data center to get data from vehicle via ZigBee and data from Cloud via Wi-Fi. Fig. 5 shows diagram of data center box. Devices used in the Data center box are ZigBee, Wi-Fi module- Node MCU, Bluetooth Module. Bluetooth module and the ZigBee are connected to one Node MCU via two separate UART ports.

Node MCU is development board of esp8266, 32-bit microcontroller insidewith $160 \mathrm{MHz}$ clock frequency. Node MCU fetches the data coordinates from the Firebase cloud storage and sends to the computer via Bluetooth.

\section{Published By:}

Blue Eyes Intelligence Engineering and Sciences Publication

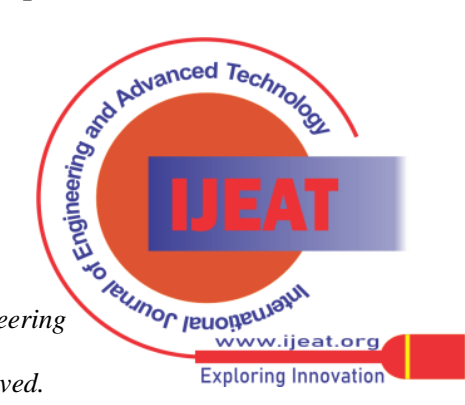


Data is received from the vehicle via ZigBee and transmitted to the computer via the Bluetooth module. Further that data is processed by software in computer.

ZigBee operates on line-of-sight principle i.e. the antenna must be in sight of at least one of vehicle antennas. So, this box must not be in close room, it must be outside the room with some height of 50-100 meter. The data center box can be connected to computer in data center by with wire or wirelessly with Bluetooth. Node MCU has serial port but for serial communication wire length must be less than $5 \mathrm{~m}$ so it is not practical. Therefore, we use Bluetooth, wireless protocol which has $100 \mathrm{~m}$ range and data transfer speed of 3-5 Mbps.

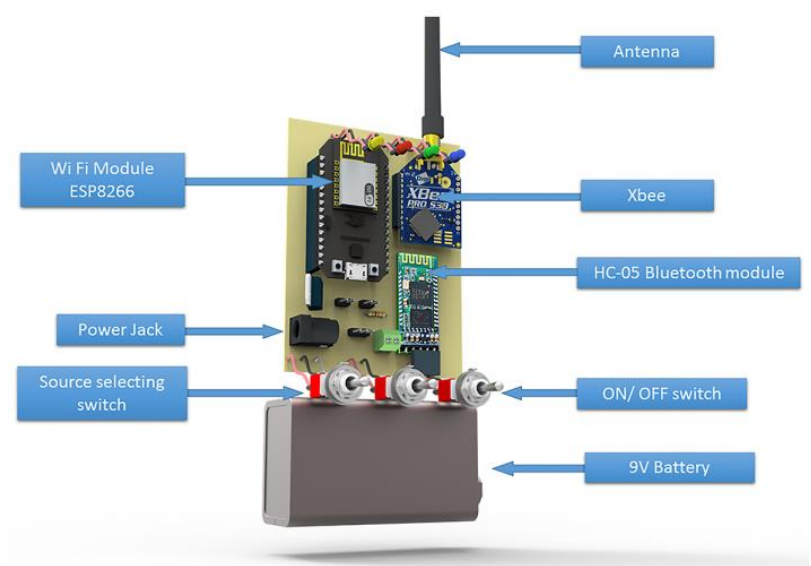

Fig. 5. Data Center Box

\section{Infrastructure Module}

Here the infrastructure means roadside infrastructures e.g. sensors, access points and centralized servers.

\section{Collection Box}

Fig. 6 shows the collection box. This box is designed for two functions, automatic toll collection and rule violation amount collection. There is a button to switch between these functions. In toll plaza, this box is mounted at a height of $10 \mathrm{~m}$. This box has IR transmitter and IR receiver (TSOP). IR transmitter transmits its ID continuously. When it is received by IR receiver of vehicle passing underneath it, IR transmitter of vehicle sends vehicle number which is received by TSOP of collection box. Wi-Fi module of collection box gets the account details of that vehicle from cloud and collects the toll amount from that account.

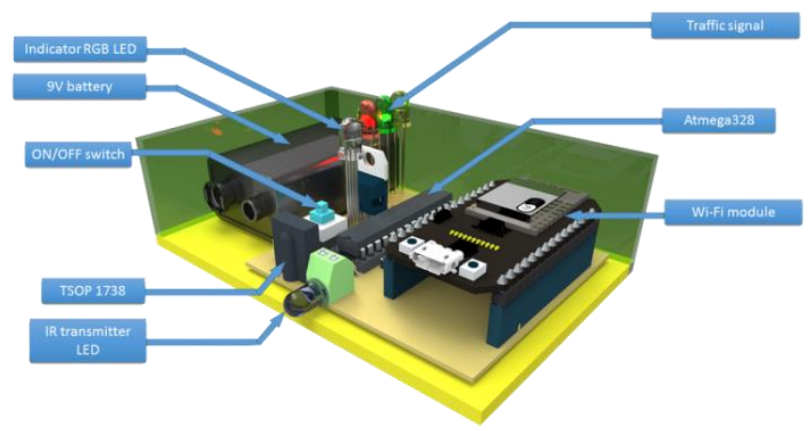

Fig. 6. Collection Box

For rule violation amount collection this box is mounted on traffic signal. It works in similar fashion as the box at toll plaza only difference is that it starts sending its ID only when the traffic signal is red to catch the vehicle, violating traffic rule. When signal is yellow or green, transmitter does not transmit signal.

\section{Cloud}

Firebase cloud is used for saving data online. Fig. 7 shows a cloud database structure for our system. After creating new project at firebase, it gives one URL, which is address of that Database [6]. Wi-Fi modules in Data center box and collection box use that URL to upload and download data. Data on firebase is saved in JavaScript Object Notation (JSON), a minimal, readable format for structuring data. Data is saved in parent child hierarchy as show in fig.7. History of any vehicle can be read from the cloud using datacenter software

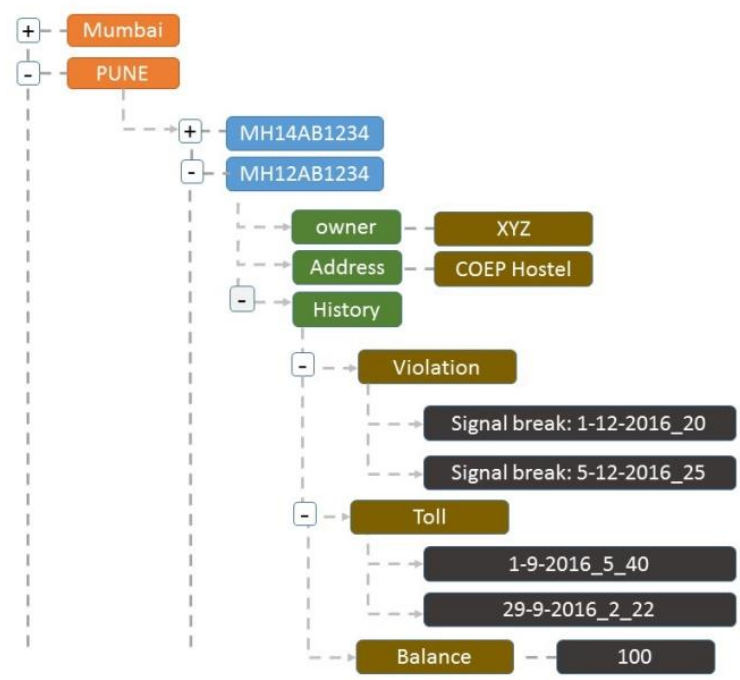

Fig. 7. Cloud database structure

\section{Networking and Protocols}

All the vehicles in SAVM system are connected with each other and data center using Zigbee in mesh topology. All ZigBees except Data center Zigbee are configured as routers, so messages generated by one vehicle will pass in mesh until it reaches the data centre. There are two ways to pass the data between the vehicle and the data centre. One is through the Firebase cloud, and the other one is through the Zigbee network. When vehicle is in city, it will be in the range of data centre directly or indirectly through mesh network. But if the vehicle is not in the range of the data centre, it can still be connected to the data centre via another vehicle which isin the data center's range. There is provision to integrate client's mobile in the system. In that case, mobile application will be connected to ZigBee box via Bluetooth and ZigBee box will upload vehicle data such as location co-ordinates, accident alert, and emergency message to Firebase account of respective vehicle.

The location coordinates can be fetched from the cloud server in the pit using Node MCU ESP8266 Wi-Fi module which is connected to an internet connection constantly and then these coordinates can be sent to the computer/laptop using the Bluetooth module.

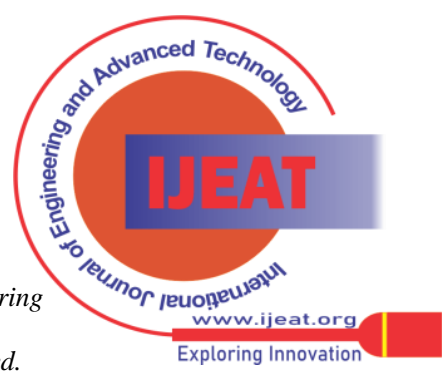


For communication between various nodes protocols are customized. The string format for them have been defined like 'STPG_MH12AB3456_18.345678,73.851234='. In this string 'STP' stands for Smart Trans-Portation, ' $G$ ' means this string contains GPS data, 'MH12AB1234' is a vehicle number, ' $18.345678,73.851234$ ' is string containing latitude and longitude separated by comma. ' $=$ ' denotes end of string.

Table-I shows the string format for traffic rule violation communication between vehicle and collection box. Table-II string format for toll collection communication between vehicle and collection box shows and Table-III gives the string format for communicating different messages between vehicle and data center software.

TABLE-I. VEHICLE-COLLECTION BOX STRING FORMAT For TRAFFiC RULE VIOLATION

\begin{tabular}{|c|c|c|}
\hline String format & Sender & Message \\
\hline $\begin{array}{c}\text { RULE_MH12A } \\
\text { B3456 }=\end{array}$ & $\begin{array}{c}\text { from vehicle to } \\
\text { Collection box }\end{array}$ & $\begin{array}{c}\text { send vehicle } \\
\text { no. }\end{array}$ \\
\hline $\begin{array}{c}\text { RULE_RL1245 } \\
12=\end{array}$ & $\begin{array}{c}\text { from Collection box } \\
\text { to vehicle }\end{array}$ & Send ID to car \\
\hline
\end{tabular}

TABLE-II. VEHICLE-COLLECTION BOX STRING FORMATTOLL COLLECTION COMMUNICATION PRotocols

\begin{tabular}{|c|c|c|}
\hline String format & Sender & Message \\
\hline $\begin{array}{c}\text { TOLL_TL1245 } \\
12=\end{array}$ & $\begin{array}{c}\text { from Collection } \\
\text { box to vehicle }\end{array}$ & Send ID to car \\
\hline $\begin{array}{c}\text { TOLL_MH12A } \\
\text { B3456_CAR= }\end{array}$ & $\begin{array}{c}\text { from vehicle to } \\
\text { Collection box }\end{array}$ & $\begin{array}{c}\text { Send vehicle no. }+ \\
\text { vehicle type }\end{array}$ \\
\hline
\end{tabular}

\section{TABLE-III. SOFTWARE-VEHICLE STRING FORMAT}

\begin{tabular}{|c|c|c|}
\hline String format & Sender & Message \\
\hline $\begin{array}{c}\text { STPG_MH12A } \\
\text { B3456_18.3456 } \\
\text { 78,73.851234= }\end{array}$ & from car & GPS position \\
\hline $\begin{array}{c}\text { STPP_MH12A } \\
\text { B3456_12= }\end{array}$ & from car & pollution scale \\
\hline $\begin{array}{c}\text { STPE_MH12A } \\
\text { B3456= }\end{array}$ & from car & EMERGENCY \\
\hline $\begin{array}{c}\text { STPA_MH12A } \\
\text { B3456= }\end{array}$ & from car & accident \\
\hline $\begin{array}{c}\text { STPM_MH12A } \\
\text { B3456_Message } \\
=\end{array}$ & from software & message \\
\hline $\begin{array}{c}\text { STPT_MH12A } \\
\text { B3456= }\end{array}$ & from software & track \\
\hline $\begin{array}{c}\text { STPR_MH12A } \\
\text { B3456_2= }\end{array}$ & from firebase & Rule No. \\
\hline
\end{tabular}

\section{CALCULATION}

\section{E. Maximum vehicle for one Data Center}

Maximum data rate of XBEE (XBEE is ZigBee manufactured by Digi International) is $156 \mathrm{kbps}$ [2].

$$
\begin{aligned}
\text { Data rate }= & 156 \mathrm{kbps}=19.5 \mathrm{kBps} \\
& =1604800 * 1024 \text { Bytes per day }
\end{aligned}
$$

Table-IV shows the amount of data used by one vehicle in one day. This is the maximum data that can be used by a vehicle in one day. Using the data rate of ZigBee and data used by one vehicle, we can calculate maximum number of vehicles accommodated in the data center.

$$
\begin{aligned}
\text { Max. no. of vehicles in a data center }= & \frac{1604800 \times 1024}{51874.665} \\
& =31,678 \text { vehicles }
\end{aligned}
$$

So, nearly $31.5 \mathrm{k}$ vehicles can be handled by one data center. This number is dependent on the ZigBee data rate. Thus we can increase the capacity of data center by using a ZigBee of higher data rate.

TABLE-IV. Data Used By Single Vehicle Per Day

\begin{tabular}{|c|c|c|}
\hline Data & No. of bytes & $\begin{array}{c}\text { No. of bytes per } \\
\text { day }\end{array}$ \\
\hline Location & 36 bytes per 1 minute & 51840 \\
\hline $\begin{array}{c}\text { Pollution } \\
\text { level }\end{array}$ & 19 bytes per day & 19 \\
\hline Emergency & 16 bytes per 6 month & 0.0879 \\
\hline Accident & 16 bytes per year[7] & 0.04383 \\
\hline Message & 30 bytes per 2 day & 15 \\
\hline Tracking & 16 bytes per month & 0.5333 \\
\hline & Total & $\mathbf{5 1 8 7 4 . 6 6 5}$ \\
\hline
\end{tabular}

\section{F. Maximum Speed of vehicle under collection box}

Angle of transmission for IR LED is $10^{\circ}$ and for TSOP angle of reception is $10^{\circ}$. So IR communication will be possible till angle of vehicle is less than $20^{\circ}$ [7]. Maximum distance of receiver is $10 \mathrm{~m}$. This is shown in fig. 8 .

$$
\begin{aligned}
\text { Max. distance of communication } & =2 \times 10 \sin \theta \\
& =3.472 \mathrm{~m}
\end{aligned}
$$

Time require for communication is $85 \mathrm{mS}$, therefore

Max. speed of vehicle $=$

$$
\frac{3.473 \times 10^{-3} \times 3600}{85 \times 10^{-3}}
$$

$$
=147 \mathrm{~km} / \mathrm{hr}
$$

Hence vehicle with speed lower than $147 \mathrm{~km} / \mathrm{hr}$ can be detected by collection box.

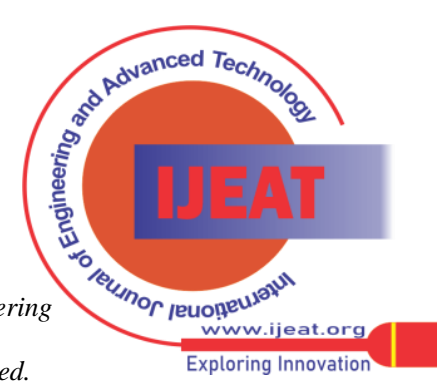




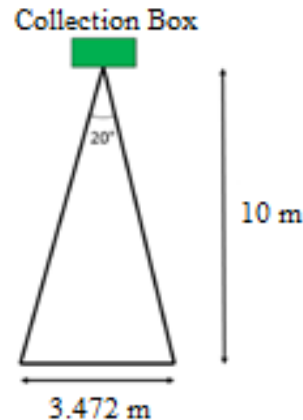

Fig. 8. Geometry for maximum distance calculation

\section{TEST AND RESULTS}

All the electronic circuits are designed and fabricated in-house at FAB LAB in college of Engineering Pune. PCBs are designed using EAGLE and then fabricated using conventional method. Outer casing of each box is made up of $3 \mathrm{~mm}$ acrylic sheet as acrylic has high strength to weight ratio. After fabrication, the complete system is deployed and tested.

Fig. 9 Shows the testing of the SVAM system. For testing the ZigBee box is mounted on two wheeler carriage. Ultra sonic distance sensor mounted on backside as well as on front side.

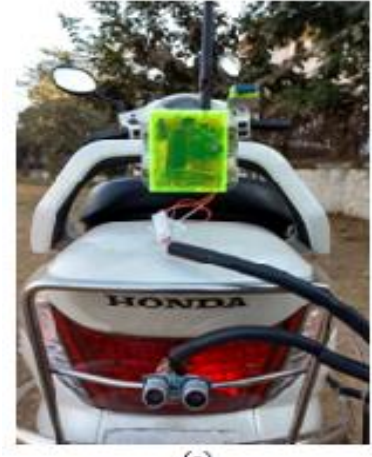

(a)

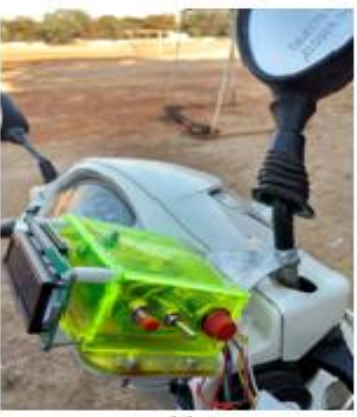

(c)

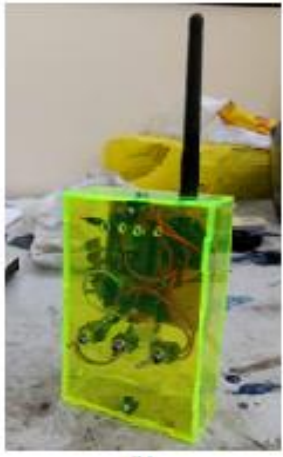

(b)

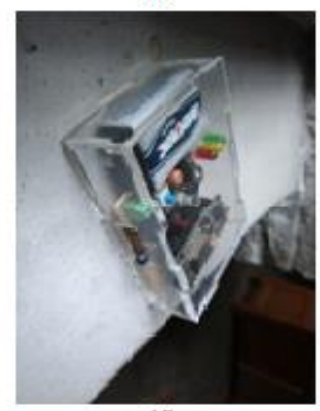

(d)
Figure 9. Fabricated and implemented (a) ZigBee Box (b) Data Center Box (c) GPS Box and (d) Collection Box

Table- $\mathrm{V}$ shows the power consumed by different circuits.

TABLE-V. POWER CONSUMED BY CIRCUITS

\begin{tabular}{|c|c|c|c|}
\hline Circuit & $\begin{array}{c}\text { Current } \\
(\mathrm{mA})\end{array}$ & $\begin{array}{c}\text { Voltage } \\
(\mathrm{V})\end{array}$ & Power \\
\hline GPS box & 100 & 5 & $0.5 \mathrm{~W}$ \\
\hline ZigBee box & 120 & 5 & $0.6 \mathrm{~W}$ \\
\hline Data Center box & 300 & 9 & $2.7 \mathrm{~W}$ \\
\hline Collection box & 300 & 9 & $2.7 \mathrm{~W}$ \\
\hline
\end{tabular}

\section{CONCLUSION}

We have successfully implemented a prototype of smart transportation system which is quite effective and efficient. The use of this system with sufficiently large number of vehicles can definitely reduce the traffic related issues to a large extent.

\section{APPENDIX}

Video of working prototype:

https://drive.google.com/file/d/0BwUbrrz81ugba3dyMWE1 SEITUVE/view?usp=sharing

\section{ACKNOWLEDGMENT}

Authors would like to thank to Department of Electronics and telecommunication, College of Engineering, Pune for funding and supporting this project work.

\section{REFERENCES}

1. Maimaris, Athanasios, and George Papageorgiou. "A review of Intelligent Transportation Systems from a communications technology perspective." Intelligent Transportation Systems (ITSC), 2016 IEEE 19th International Conference on. IEEE, 2016.

2. sparkfun.com, "Reference for specification of XBEE." 2016. [Online] 3. ieee.org, "Reference for GPS module mounting." 2016. [Online].

4. processing.org, "Reference for processing language." 2016. [Online].

5. lagers.org.uk, "Reference for G4P GUI library." 2014. [Online].

6. firebase.google.com, "Reference for firebase cloud programming." 2016. [Online].

7. Wern-YarngShieh, Chen-Chien (James) Hsu, Shen-Lung Tung, Po-Wen Lu, Ti-Ho Wang, Shyang-LihChang, “ Design of Infrared Electronic-Toll-Collection Systems With Extended Communication Areas and Performance of Data Transmission." IEEE Transactions On Intelligent Transportation Systems, Vol. 12, No. 1, March 2011.

\section{AUTHORS PROFILE}

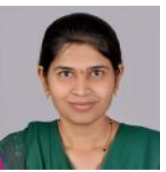

Dr. Deeplaxmi V. Niture has received the B. Tech and M. Tech degree in Electronics and Telecommunication Engineering from Dr. Babasaheb Ambedkar Technological University (Dr. B. A. T. U), Lonere, India in 2003, and in 2006 respectively. She has completed her Ph.D. in Electronics and Telecommunication Engineering from the Savitribai Phule University of Pune, Maharashtra, India in 2021. Presently she is working as assistant professor in College of Engineering Pune (COEP). She has authored several journal and conference papers. Her research interests include Microstrip Antennas, Reconfigurable antennas, RF and Microwave Circuits and Internet of Things.

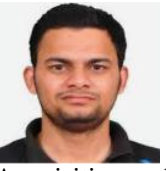

Vivekanand Dhakane has completed B. Tech in Electronics and Telecommunication Engineering from College of Engineering Pune in 2017 and currently pursuing M. Tech in Solid State Devices at Indian Institute of Technology, Bombay. His areas of interest are Data Acquisition and Automotive Electronics.

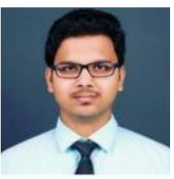

Piyush Jawalkar has completed B. Tech in Electronics and Telecommunication Engineering from College of Engineering Pune (COEP) in 2017 and currently working Credit Suisse. His area of interest is Electronics Circuit Design.

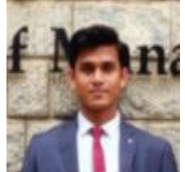

Ankit Bamnote has completed B. Tech in Electronics and Telecommunication Engineering from College of Engineering Pune (COEP) in 2017 and MBA from India Institute of Management, Bangalore in 2021. Presently he is working as Product Manager at Sprinklr.

Published By:

Blue Eyes Intelligence Engineering and Sciences Publication

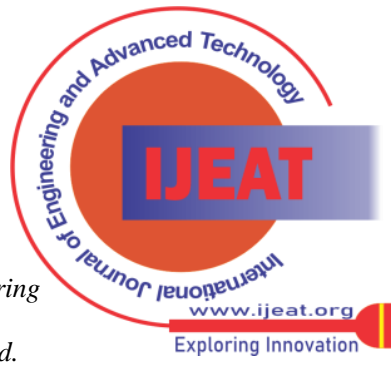

